\title{
Interstitial lung disease in a baby with a de novo mutation in the SFTPC gene
}

\author{
F. Brasch*,\#, M. Griese*, , M. Tredano ${ }^{+}$, G. Johnen\#, M. Ochs ${ }^{\S}$, C. Rieger ${ }^{f}$, S. Mulugeta**, \\ K.M. Müller", M. Bahuau ${ }^{+}$, M.F. Beers**
}

Interstitial lung disease in a baby with a de novo mutation in the SFTPC gene. F. Brasch, M. Griese, M. Tredano, G. Johnen, M. Ochs, C. Rieger, S. Mulugeta, K.M. Müller, M. Bahuau, M.F. Beers. C) ERS Journals Ltd 2004.

ABSTRACT: Mutations in the surfactant protein C gene (SFTPC) were recently reported in patients with interstitial lung disease.

In a 13-month-old infant with severe respiratory insufficiency, a lung biopsy elicited combined histological patterns of nonspecific interstitial pneumonia and pulmonary alveolar proteinosis. Immunohistochemical and biochemical analyses showed an intraalveolar accumulation of surfactant protein (SP)-A, precursors of SP-B, mature SP-B, aberrantly processed proSP-C, as well as mono- and dimeric SP-C.

Sequencing of genomic DNA detected a de novo heterozygous missense mutation of the SFTPC gene (g.1286T $>C$ ) resulting in a substitution of threonine for isoleucine (I73T) in the C-terminal propeptide. At the ultrastructural level, abnormal transport vesicles were detected in type-II pneumocytes. Fusion proteins, consisting of enhanced green fluorescent protein and wild-type or mutant proSP-C, were used to evaluate protein trafficking in vitro. In contrast to wild-type proSP-C, mutant proSP-C was routed to early endosomes when transfected into A549 epithelial cells.

In contrast to previously reported mutations, the I73T represents a new class of surfactant protein $\mathrm{C}$ gene mutations, which is marked by a distinct trafficking, processing, palmitoylation, and secretion of the mutant and wild-type surfactant protein C. This report heralds the emerging diversity of phenotypes associated with the expression of mutant surfactant $\mathbf{C}$ proteins.

Eur Respir J 2004; 24: 30-39.

\begin{abstract}
\#Institute of Pathology, University Hospital "Bergmannsheil", and 'Children's Hospital, Ruhr-University, Bochum, 'Dr. von Haunersches Children's Hospital, Ludwig-MaximiliansUniversity, Munich, and Division of Electron Microscopy, Dept of Anatomy, University of Göttingen, Göttingen, Germany. ${ }^{+}$Service de Biochimie et Biologie Moléculaire, Hôpital d'Enfants Armand-Trousseau (AP-HP), Paris, France. **Lung Epithelial Cell Biology Laboratories, Pulmonary and Critical Care Division, Dept of Medicine, University of Pennsylvania School of Medicine, Philadelphia, PA, USA.
\end{abstract}

Correspondence: F. Brasch, Institute of Pathology, University Hospital "Bergmannsheil", Bürkle-de-la-Camp Platz 1, D-44789 Bochum, Germany.

Fax: 49 234/3026671

E-mail: Frank.E.Brasch@ruhr-uni-bochum.de

Keywords: Interstitial lung disease, nonspecific interstitial pneumonia, pulmonary alveolar proteinosis, surfactant protein $\mathrm{C}$, surfactant protein $\mathrm{C}$ gene

Received: January 12004

Accepted after revision: March 232004

Major funding was provided as a gift from M. Pressac, Pharm.D. Additional support came from NIH HL-19737, NIH HL-074064, and P50-HL-56401 (M.F. Beers) and DFG Gr 970/7-1 (M. Griese).

*These authors contributed equally to this work.
Pulmonary surfactant is a complex mixture of lipids and hydrophilic, as well as hydrophobic, proteins that reduces surface tension by forming a surface-active film at the air/ liquid interface of the alveolus. Four surfactant-associated proteins have been identified: surfactant protein (SP)-A, SP-B, SP-C and SP-D [1]. The hydrophobic SP-B and SP-C play an essential role in the metabolism and dynamics of the lipids of pulmonary surfactant by promoting the rapid adsorption of phospholipids into the monolayer and the stabilisation of the surfactant film [2]. Hereditary SP-B deficiency was the first reported genetic cause of fatal respiratory distress syndrome in newborns [3]. As an eosinophilic and periodic acid Schiff (PAS)-positive material was found in the alveoli, hereditary SP-B deficiency was initially described as "congenital alveolar proteinosis syndrome" [3, 4]. However, in contrast to "cryptogenic" congenital, idiopathic and secondary pulmonary alveolar proteinosis (PAP), hereditary SP-B deficiency is associated with SP-B gene (SFTPB) mutations and is characterised by a complete or incomplete deficiency of SP-B, an aberrant processing of proSP-C and a lack of mature SP-C [5-7].
PAP, first described in 1958 by Rosen et al. [8], is a distinct lung disorder characterised histologically by an intra-alveolar accumulation of granular eosinophilic and PAS-positive material. PAP represents a heterogeneous group of congenital or acquired lung diseases in newborns, infants and adults Idiopathic PAP in adults is the most common form and a neutralising antibody against granulocyte macrophage-colony stimulating factor (GM-CSF) was characterised recently [9] Secondary alveolar proteinosis has been reported in several clinical settings: it comprises aberrant responses to infection and inhalation of minerals or chemicals; or it may be associated with underlying diseases, such as lymphomas, and acute and chronic leukaemias [10].

Idiopathic interstitial pneumonias (IIP) represent a heterogeneous group of non-neoplastic lung disorders that include: usual interstitial pneumonia (UIP); nonspecific interstitial pneumonia (NSIP); cryptogenic organising pneumonia; acute interstitial pneumonia; respiratory bronchiolitis-associated interstitial lung disease; desquamative interstitial pneumonia (DIP); and lymphocytic interstitial pneumonia (LIP) [11]. 
Familial forms of UIP, DIP and LIP have been described. Although it is estimated that $0.5-2.2 \%$ of interstitial pneumonias have some genetic basis [12], virtually nothing is known of the specific genes whose mutations underlie IIP.

Recently, two heterozygous mutations of the SFTPC gene were described in two families, which showed a lack of mature SP-C and interstitial lung disease [13, 14]. In the present report, the authors describe a de novo heterozygous SFTPC mutation in a full-term baby boy, which is associated with combined histological patterns of NSIP and PAP. Detailed biochemical characterisation indicates that expression of mutant proSP-C results in abnormal proprotein trafficking, leading to an accumulation of aberrantly processed proSP-C in the alveoli.

\section{Materials and methods}

\section{Study subject}

The index patient was a full-term baby boy born after an uneventful pregnancy. The parents reported slight dyspnoea and tachypnoea at the age of 1 month. At 3 months, he developed recurrent bronchitis. As the dyspnoea worsened, the patient showed failure to thrive, and a need for supplementary oxygen developed at the age of 9 months. Screening for metabolic, infectious and immunological aetiologies was negative. As the need for supplementary oxygen increased and chest radiographs showed persistent diffuse bilateral pulmonary infiltrates, the boy was transferred to a university hospital at the age of 11 months. A SFTPB mutation was excluded by SFTPB sequencing [5]. Recently, a neutralising antibody against GM-CSF in adult patients with idiopathic PAP was characterised and it has also been shown that such auto-antibodies are diagnostic for autoimmune (idiopathic) PAP $[9,15,16]$. Auto-antibodies against GM-CSF were not detected in serum or in bronchoalveolar lavage (BAL) effluents of the index patient. FACS analysis of peripheral blood mononucleated cells revealed the presence of functionally active GM-CSF receptors (CD116; S. Burdach, MartinLuther University, Halle, Germany, personal communication). Heart, immunological, metabolic and infectious diseases (pneumocystis, mycoplasma, chlamydia and viruses) were systematically excluded. Since his respiratory function worsened, an open lung biopsy was performed when the child was 13 months old.

\section{Mutation analysis by gene sequencing}

SFTPC exons 1-6 were polymerase chain reaction (PCR)amplified based on the sequence provided by GLASSER et al. [17] (GenBank accession \#J03890.1) in the proband and parents. The primers used for detecting the mutation (exon 3) are given in table 1 . The purified PCR products served as templates in the sequencing reaction, using the ready reaction dye terminator cycle sequencing kit with AmpliTaq $\mathbb{R}$ DNA polymerase, FS (PE Biosystems, Foster City, CA, USA), with forward and reverse PCR oligonucleotides used as extension primers. Extension products were analysed using the ABI

Table 1.-Polymerase chain reaction primers: exon 3 amplification

Primer location Primers (5' to $\left.3^{\prime}\right)$

Intron 2 Exon $3(\mathrm{~F})$ : AAGCGCATTTGAGTACAGAGG Intron 3 Exon $3(\mathrm{R})$ : ATGGATGTGGAATGAAAGTGG
PRISM $\mathbb{R} 310$ Genetic Analyzer (PE Biosystems) and the Sequence Analysis 3.0 software (PE Biosystems).

\section{Mutation screening by endonuclease cleavage}

Since the newly described g.1286T $>C$ (I73T) lesion generated a recognition site for restriction endonuclease BsrI, this enzyme was used for confirmatory purposes in the proband and for rapidly screening the familial DNAs, as well as a cohort of 100 unrelated ethnically-matched control chromosomes. To this end, DNAs were PCR-amplified using primers exon $3(\mathrm{~F})$ and $(\mathrm{R})$ (table 1), and amplification products were restricted with BsrI (New England Biolabs, Beverly, MA, USA) for $5 \mathrm{~h}$ at $65^{\circ} \mathrm{C}$ and size-fractionated through $10 \%$ polyacrylamide gels.

\section{Assessment of relatedness}

Relatedness was assessed through the genotyping at eight highly polymorphic DNA markers: D7S486, D8S285, D9S171, IFNA1, D12S391, D18S1147, D20S194 and D21S226. Fragment analysis was performed on an ABI PRISM $\mathbb{R} 3100$ Genetic Analyzer (PE Biosystems) with internal GeneScan ${ }^{\mathrm{TM}}$ 350 TAMRA $^{\text {TM }}$ Size Standard (PE Biosystems) using the

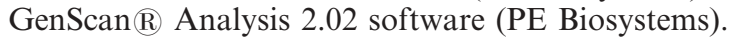

\section{Phase determination}

Haplotypes were determined based on a series of eight intragenic (substitution) single-nucleotide polymorphisms (SNPs) and one (insertion/deletion) SNP (data not shown). The phase was then established between the mutation and the most adjacent SNPs that happened to be polymorphic in the proband. In particular, forward allele-restricted PCR primers were designed for the 745T/C SNP, for DNA amplification with a common reverse primer located downstream of intron 3 (table 2). After assessing amplification specificity, PCR products were purified and submitted to sequence analysis, as described previously and using the common reverse PCR oligonucleotide as an extension primer.

\section{Transmission electron microscopy}

For transmission electron microscopy studies, lung samples were fixed by immersion in $1 \%$ glutaraldehyde and $4 \%$ paraformaldehyde in $0.1 \mathrm{M}$ phosphate buffer, post-fixed in $2 \%$ osmium tetroxide, and embedded in Epon. Ultra-thin sections were contrasted with uranyl acetate followed by lead citrate, and were examined with a Zeiss 900 electron microscope (Carl Zeiss, Oberkochen, Germany).

\section{Antisera}

Polyclonal antisera or monoclonal antibodies against SP-A (PE-10), SP-B, leukocyte common antigen (LCA), CD3, CD4, CD8, CD86, thyroid transcription factor (TTF)-1 and vimentin were purchased from Dako (Glostrup, Denmark), Chemicon (Temecula, CA, USA), Novocastra (Newcastleupon-Tyne, UK) and Neo Markers (Westinghouse, CA, USA). A monoclonal antibody against human SP-B was kindly donated by Y. Suzuki (Dept of Ultrastructural Research, Kyoto University, Japan). Polyclonal rabbit antiserum against proSP-B (anti-proSP-B) was kindly provided 
Table 2. - Polymerase chain reaction primers: phase determination using allele-specific primers

\begin{tabular}{lccr}
\hline Nucleotide variants & Primer location & Primers $\left(5^{\prime}\right.$ to $\left.3^{\prime}\right)$ & \\
\hline 745T/C (intron 1) & Intron 1 & Allele-specific primers & 745T (F): ATGACCTCATGCCTGTCTCCT \\
T1286C (exon 3) & Intron 3 & Common primer & 745C (F): ATGACCTCATGCCTGTCTCCE \\
\hline
\end{tabular}

\#: allele-specific nucleotides are underlined.

by S. Hawgood (Dept of Pediatrics and Cardiovascular Research Institute, University of California, San Francisco, CA, USA). The polyclonal antibodies against the $\mathrm{N}$-terminal propeptide domain $\left(\mathrm{E}^{11}-\mathrm{R}^{23}=\right.$ anti-NPROSP-C) and the distal C-terminal propeptide domain $\left(\mathrm{G}^{162}-\mathrm{G}^{174}=\right.$ anti-CTERMSP-C) of human proSP-C have been characterised previously [18, 19]. A polyclonal antiserum against mature SP-C was kindly provided by W. Steinhilber (Avanta Pharmaceuticals, Konstanz, Germany).

\section{Immunohistochemistry}

For immunohistochemistry, lung biopsy specimens were fixed by immersion in $4 \%$ buffered formaldehyde. Immunostaining was carried out using the alkaline-phosphatase method as previously described in detail [20].

\section{Controls}

For immunostaining analyses, lung biopsy specimens from nine babies, who had undergone cardio-thoracic surgery due to congenital heart defects, but without secondary lung disease (pulmonary hypertension), were used as normal controls. To further compare the index case with other causes of respiratory distress, additional controls consisted of biopsy specimens from three newborns with complete SP-B deficiency due to 12 ins 2 or 122delT SFTPB mutations [21], and from two full-term babies with "cryptogenic" congenital alveolar proteinosis.

\section{Western blot analysis of bronchoalveolar lavage fluid}

The fluid recovered from BAL was centrifuged at $200-300 \times g$ for $15 \mathrm{~min}$ to remove cells, and aliquots of the supernatant were stored at $-80^{\circ} \mathrm{C}$. Immediately before blotting, aliquots were thawed and separated by gel electrophoresis on pre-cast $4-12 \%$ NuPage Bis-Tris polyacrylamide gels (Novex/Invitrogen, Carlsbad, CA, USA). Immunoblotting with antisera against proSP-B, SP-B, NPROSP-C, CTERMSP-C and SP-C was performed as previously described [20].

\section{ELISA for surfactant protein $A$}

The assay was performed as previously described [22].

\section{In vitro analysis of pro-surfactant protein $C^{I 73 T}$ expression}

Constructs for chimeric fusion proteins consisting of enhanced green fluorescence protein (EGFP) and either wild type proSP-C (EGFP/proSP-C $\left.{ }^{1-197}\right)$ or the mutant protein (EGFP/proSP-C ${ }^{\text {I73T }}$ ) were generated by PCR using full-length human SFTPC cDNA (generous gift from P. Ballard, Pediatrics, University of Pennsylvania/Children's Hospital of Philadelphia, PA, USA) or cDNA containing the mutation $(\mathrm{g} .1286 \mathrm{~T}>\mathrm{C} \quad(\mathrm{I} 73 \mathrm{~T}))$ in the C-terminal propeptide as previously described [23, 24]. Automated DNA sequencing in both directions failed to detect in vitro nucleotide mutations in wild-type or mutant proSP-C constructs. A549 cells (American Type Culture Collection, Manasas, VA, USA) were transiently transfected with EGFP/proSP-C ${ }^{1-197}$ or EGFP/ proSP-C ${ }^{173 T}$ constructs, as previously described [25]. For immunolabelling studies, fixation by immersion of coverslips in $4 \%$ paraformaldehyde and permeabilisation of cells with Triton X-100 buffers (Sigma, St Louis, MO, USA) was followed by incubation with primary antisera for $1 \mathrm{~h}$ at room temperature, followed by either secondary goat anti-mouse immunoglobulin (Ig)G monoclonal or secondary goat antirabbit IgG polyclonal antisera.

\section{Results}

\section{Light and transmission electron microscopy}

Histological examination of the lung biopsy from the index patient showed an intra-alveolar accumulation of a finely granular eosinophilic and PAS-positive material, as well as a thickening of alveolar septa (fig. 1a). Immunostaining for TTF-1 (nuclear red staining) revealed a marked hyperplasia of type-II pneumocytes (fig. 1b). Spindle-shaped cells in alveolar septa were strongly stained for vimentin, suggesting that the increased cellularity was mainly due to the proliferation of mesenchymal cells (fig. 1c). Furthermore, focally accumulated alveolar macrophages were stained for vimentin (fig. 1c) and CD68 (not shown). Immunostaining for LCA revealed the presence of a mild interstitial chronic inflammation (fig. 1d). Most of the leukocytes were CD3- and CD4positive T-lymphocytes (not shown). At the ultrastructural level, hyperplasia of type-II pneumocytes was confirmed and abnormal vesicular organelles with an electron dense core were detected (fig. 1e). These abnormal organelles were also found in the process of secretion (fig. 1f).

\section{Mutation analysis by gene sequencing and screening by endonuclease cleavage}

Sequence analysis, as performed on the proband's DNA, yielded a heterozygous $\mathrm{T}>\mathrm{C}$ transition in exon 3 of the gene in position 1286, with regard to the reference genomic sequence of GLASSER et al. [17] (data not shown). For reasons of consistency with a previously described SFTPC mutation [13] and with the nomenclature system as agreed upon for reporting mutations in SFTPB (MIM 178640) [5], the transcription start site was denoted +1 , and not the $\mathrm{A}$ of the ATG of the initiator Met codon. This mutation accounted for the substitution of a threonine for a highly conserved isoleucine in position 73. Therefore, the mutation may report as g.1286T $>$ C or I73T. Using restriction by the endonuclease BsrI, the mutation was not found in the parents (fig. 2), nor was it in 100 unrelated Caucasian individuals (data not shown), suggesting its de novo occurrence. Sequence analysis in the parents confirmed this result (data not shown). 


\section{Assessment of relatedness and phase determination}

Genotyping of the nuclear family, with eight highly polymorphic markers scattered throughout the human genome, yielded no evidence of nonpaternity or nonmaternity (table 3), further supporting the notion that the mutation was inherited de novo. Using nine physically linked bi-allelic markers (data not shown), all intragenic to SFTPC, the two polymorphic markers that happened to be the most adjacent to the mutation were 745T/C upstream (intron 1) and 2182A/G downstream (exon 5) (fig. 2). Phase determination using forward allelerestricted PCR primers designed for $745 \mathrm{~T} / \mathrm{C}$ and a common reverse primer located downstream of intron 3 indicated that the mutation occurred on the paternal allele (fig. 3).

\section{Characterisation of surfactant-associated proteins by immunochemistry and Western-blot}

Lung biopsy specimens from controls and the index patient were stained for SP-A, proSP-B, SP-B, NPROSP-C, CTERMSP-C and SP-D.
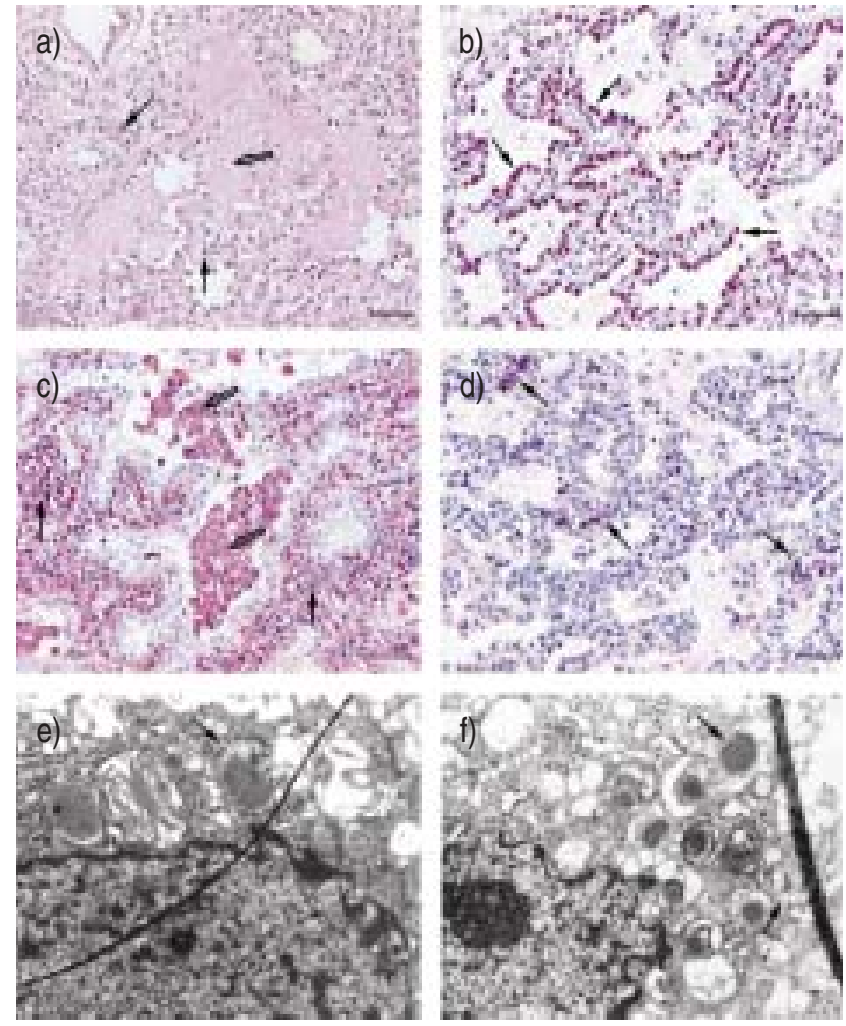

Fig. 1.- Morphological features of interstitial lung disease in the index patient. a) Histological examination of haematoxylin and eosin-stained sections showed a hyperplasia of type II pneumocytes, thickening of alveolar septa (arrow) due to spindle-shaped cells and a mild interstitial chronic inflammation (nonspecific interstitial pneumonia-like feature), as well as a finely granular eosinophilic material filling many alveoli (open arrow; pulmonary alveolar proteinosis-like feature). Immunohistochemical analysis confirmed b) a hyperplasia of thyroid transcription factor-1positive type-II pneumocytes (arrows), c) a thickening of alveolar septa mainly due to vimentin-positive mesenchymal cells (arrows) and d) a mild interstitial infiltrate of leukocyte common antigen-positive mononuclear cells (arrows). Furthermore, a focal intra-alveolar accumulation of vimentin and CD68 (not shown)-positive macrophages (open arrow) with a foamy cytoplasm was present (c). At the ultrastructural level, e) abnormal vesicular organelles (arrow) with an electron dense core were found in type-II pneumocytes, f) with some organelles (arrow) in the process of being secreted. Scale bar $=50 \mu \mathrm{m}$.
Lung biopsy specimens from controls, who had undergone cardio-thoracic surgery due to congenital heart defects, showed a moderate-to-strong staining of type-II pneumocytes for SP-A, proSP-B, SP-B, NPROSP-C, CTERMSP-C and SP-D. Furthermore, immunohistochemistry for SP-A and SP-B showed a moderate-to-strong positive staining at the alveolar epithelial surface. ProSP-B, NPROSP-C and CTERMSP-C staining were not detected in the alveolar space (not shown).

In the index patient, type-II pneumocytes stained for SP-A, proSP-B, SP-B, NPROSP-C and CTERMSP-C. In line with the strong staining of the intra-alveolar material for SP-A (fig. 4a), the SP-A level in the BAL fluid was increased to $32,000 \mu \mathrm{g} \cdot \mathrm{mL}^{-1}$ (normal: $900 \mu \mathrm{g} \cdot \mathrm{mL}^{-1}$; range: $220-9,000$ ) [26]. Furthermore, the intra-alveolar-accumulated material also stained strongly for proSP-B and CTERMSP-C (fig. 4d, m), but showed only a moderate or weak staining for SP-B and NPROSP-C, respectively (fig. 4g, j).

In lung biopsy specimens from newborns with either 121 ins 2 or 122 delT SFTPB mutations, type-II pneumocytes and the intra-alveolar-accumulated material stained strongly for SP-A and NPROSP-C (fig. 4b, k), as well as moderately



$\begin{array}{lll}\text { c) Intron 1 } & 745 \mathrm{~T} / \mathrm{C} & \mathrm{T} \\ \text { Exon 3 } & 1286 \mathrm{~T}>\mathrm{C} & \mathrm{C} \\ \text { Exon } 4 & 1704 \mathrm{C}>\mathrm{A} & \mathrm{C} \\ \text { Exon 5 } & 2182 \mathrm{~A}>\mathrm{C} & \mathrm{G}\end{array}\|\| \begin{aligned} & \mathrm{C} \\ & \mathrm{T} \\ & \mathrm{C} \\ & \mathrm{A}\end{aligned}$

Fig. 2.-Pedigree analysis. a) Upon restriction with endonuclease $B s r \mathrm{I}$, the wild-type 306-kb exon 3 polymerase chain reaction (PCR) product generates three fragments of 145,113 and $48 \mathrm{bp}$, while the mutant generates four fragments of $113,78,67$ and 48 bp. Lane 1: molecular marker 50 bp DNA Ladder (Invitrogen ${ }^{\mathrm{TM}}$, San Diego, CA, USA); Lane 2: unrestricted exon 3 PCR product generated from the proband ( $\mathbf{\square}$ in ); Lanes 3-5: exon-3 PCR product restricted with $B s r \mathrm{I}$ from the proband, father ( $\square$ in b) and mother ( $\bigcirc$ in b), respectively. The mutation present in the proband is apparently de novo. c) Haplotyping is shown with three single nucleotide polymorphisms flanking the mutation, with their respective positions with regard to the gene organisation, indicating that the mutation is of paternal origin. 
Table 3.- Relatedness test

\begin{tabular}{|c|c|c|c|c|c|c|c|c|}
\hline \multirow[t]{2}{*}{ Individual $^{\oplus}$} & \multicolumn{8}{|c|}{ Marker heterozygosity (allele size ${ }^{\#}$ ) } \\
\hline & $\begin{array}{c}\text { D8S285 } \\
(0.77)\end{array}$ & $\begin{array}{c}\text { D7S486 } \\
(0.80)\end{array}$ & $\begin{array}{c}\text { D9S171 } \\
(0.80)\end{array}$ & $\begin{array}{c}\text { IFNA1 } \\
(0.83)\end{array}$ & $\begin{array}{l}\text { D12S391 } \\
(0.90)\end{array}$ & $\begin{array}{c}\text { D18S1147 } \\
(0.85)\end{array}$ & $\begin{array}{c}\mathrm{D} 20 \mathrm{~S} 194 \\
(0.95)\end{array}$ & $\begin{array}{c}\text { D21S226 } \\
(0.59)\end{array}$ \\
\hline \multirow[t]{2}{*}{ I. 1} & 114 & 134 & 163 & 142 & 230 & 109 & 202 & 133 \\
\hline & 114 & 134 & 135 & 149 & 239 & 120 & 217 & 138 \\
\hline \multirow[t]{2}{*}{ I. 2} & 102 & 137 & 163 & 147 & 211 & 101 & 202 & 133 \\
\hline & 112 & 137 & 170 & 149 & 219 & 116 & 209 & 138 \\
\hline \multirow[t]{2}{*}{ II.1 } & 112 & 134 & 163 & 142 & 219 & 116 & 209 & 138 \\
\hline & 114 & 137 & 163 & 147 & 239 & 120 & 217 & 138 \\
\hline
\end{tabular}

Data are presented as $\mathrm{n} .{ }^{*}$ : allele sizes in bp; ${ }^{\circ}$ : according to position in pedigree (see fig. 2).

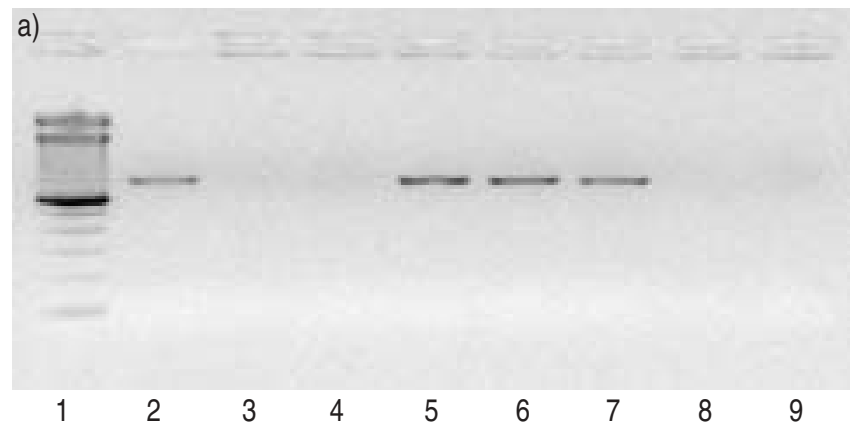

b)

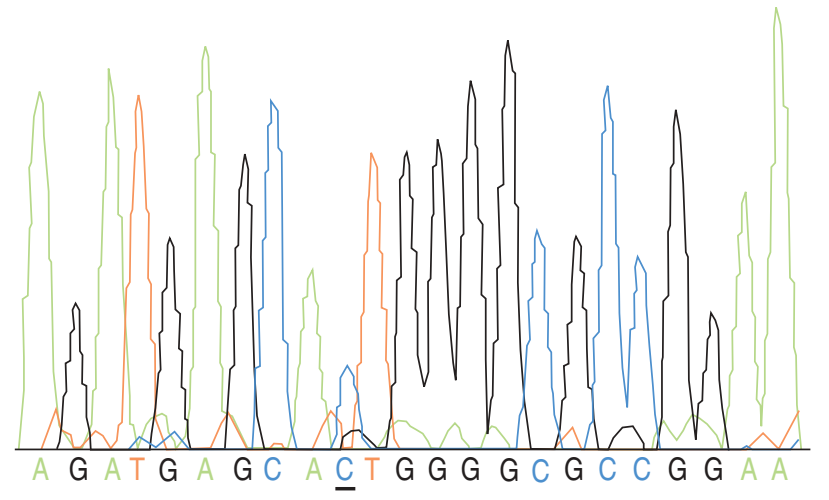

C)

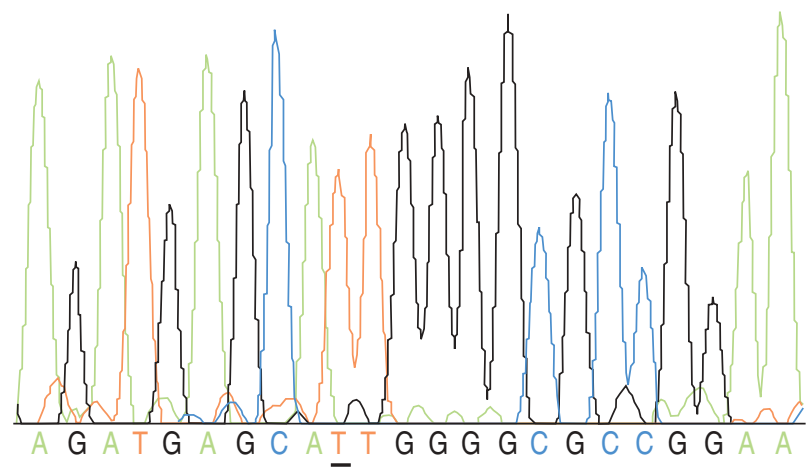

Fig. 3.-Determination of mutation phase. a) Two per cent agarose gel following electrophoresis of polymerase chain reaction (PCR) products using the common primer intron $3(\mathrm{R})$ and allele-specific primers $745 \mathrm{~T}$ (F) (even lanes) or 745C (F) (odd lanes), from the father (lanes 2, 3), mother (lanes 4, 5), proband (lanes 6, 7) and with blank samples (lanes 8, 9). Lane 1: molecular marker 100 bp DNA Ladder (Invitrogen ${ }^{\mathrm{TM}}$, San Diego, CA, USA). There is an absence of amplification product in the father when using the $745 \mathrm{C}(\mathrm{F})$ primer (lane 3 ) and in the mother when using 745T (F) (lane 4). Sequence analysis of the PCR product obtained from the proband using primers b) $745 \mathrm{~T}(\mathrm{~F})$ with intron 3 $(\mathrm{R})$ or c) $745 \mathrm{C}(\mathrm{F})$ with intron $3(\mathrm{R})$ and focusing on the mutation site. The wild-type sequence (nt. 1286T, underlined) is in cis with nt. 745C; whereas, the mutated sequence (nt. $1286 \mathrm{C}$, underlined) is in cis with nt. $745 \mathrm{~T}$, indicating that the mutation is of paternal origin. for SP-D (not shown), whilst CTERMSP-C was detectable only in type-II pneumocytes (fig. 4n). Immunostaining for proSP-B and SP-B was completely negative (fig. 4e, h).

In lung biopsies from full-term babies with cryptogenic congenital alveolar proteinosis, type-II pneumocytes and the intra-alveolar-accumulated material showed a moderate-tostrong staining for SP-A, proSP-B and SP-B (fig. 4c, f, i), but only type-II pneumocytes stained for NPROSP-C and CTERMSP-C (fig. 4l, o).

Consistent with the immunohistochemical staining pattern of the intra-alveolar-accumulated material of the index patient, anti-CTERMSP-C detected two bands at 11 and $13 \mathrm{kDa}$, whilst anti-NPROSP-C elicited only a very weakly stained band at $15 \mathrm{kDa}$ (fig. 5a, lanes 1 and 2). Anti-mature SP-C identified two bands at $4 \mathrm{kDa}$ and $8 \mathrm{kDa}$, corresponding in sizes and antigenic characteristics to mono- and dimeric SP-C (fig. 5a, lane 3). Anti-proSP-B and anti-SP-B detected a $23-\mathrm{kDa}$ precursor of SP-B and mature dimeric 18-kDa SP-B, respectively (fig. 5a, lanes 4 and 5).

\section{EGFP/proSP-C $C^{I 73 T}$ is targeted to early endosomes in A549 cells}

In order to exclude the possibility of a nonfunctional polymorphism, in vitro trafficking studies were performed, utilising lung epithelial cells transfected with expression constructs encoding fusion proteins, consisting of EGFP and either wild-type proSP-C (proSP-C ${ }^{1-197}$ ) or the I73T mutant form (proSP-C ${ }^{173 T}$ ). In control transfections using $\mathrm{EGFP} /$ proSP-C $\mathrm{C}^{1-197}$, expression of fusion protein occurred in cytosolic vesicles. In co-localisation studies, EGFP/proSP-C ${ }^{1-}$ 197 co-localised with the lysosomal marker CD63 (data not shown). Consistent with previously published results, this compartment is also early endosome antigen (EEA)1-negative, indicating that wild-type fusion protein targets to acidic lysosomal-like organelles (data not shown). In contrast, trafficking of the proSP-C ${ }^{\mathrm{I} 3 \mathrm{~T}}$ mutant was markedly different with spatial distribution of EGFP/proSP-C $C^{173 T}$ in smaller cytosolic vesicles (fig. 6). Further subcellular localisation of the mutant protein revealed partial co-localisation in EEA1positive vesicles, with a second pool of proSP-C ${ }^{173 T}$ mutant juxtapositioned with the plasma membrane that failed to stain for EEA1.

\section{Therapeutic strategy}

As interstitial lung disease and alveolar filling with proteinaceous material resulted in severe abnormalities of gas exchange, the patient was treated by repetitive whole-lung lavages, and with systemic corticosteroids and azathioprin 



Fig. 4. - Pattern of surfactant proteins in the g.1286T $>C$ (I73T) surfactant protein $C$ gene missense heterozygote (a, d, g, j, m), in a surfactant protein B gene 121ins2 homozygote (b, e, h, k, n), and in a full-term baby with cryptogenic congenital pulmonary alveolar proteinosis (PAP) (c, $\mathrm{f}, \mathrm{i}, \mathrm{l}, \mathrm{o}$ ). The intra-alveolar-accumulated material of the index patient (a) and the patients with hereditary surfactant protein (SP)-B deficiency (b), as well as cryptogenic congenital PAP (c), stained strongly for SP-A. While neither proSP-B nor SP-B were detectable in hereditary SP-B deficiency $(\mathrm{e}, \mathrm{h})$, type-II pneumocytes and the intra-alveolarly accumulated material showed a moderate to strong staining for proSP-B and SP-B in the index patient $(\mathrm{d}, \mathrm{g})$ and in cryptogenic congenital PAP ( $\mathrm{f}, \mathrm{i})$. In line with an insufficient N-terminal processing of proSP-C in hereditary SP-B deficiency, the intra-alveolar material stained strongly for NPROSP-C but not CTERMSP-C (k, n). In contrast to hereditary SP-B deficiency, the strong staining of the intra-alveolar-accumulated surfactant for CTERMSP-C $(\mathrm{m})$ but not NPROSP-C (j) provides evidence for an intra-alveolar accumulation of C-terminal insufficiently processed proSP-C in the index patient. In cryptogenic congenital PAP, only type-II pneumocytes stained for NPROSP-C and CTERMSP-C (1, o). Scale bar=50 $\mu \mathrm{m}$. 
a)

$23 \mathrm{kDa}$

$18 \mathrm{kDa}$

$15 \mathrm{kDa}$

$13 \mathrm{kDa}$

$11 \mathrm{kDa}$

$8 \mathrm{kDa}$

$4 \mathrm{kDa}$

1

2

b)

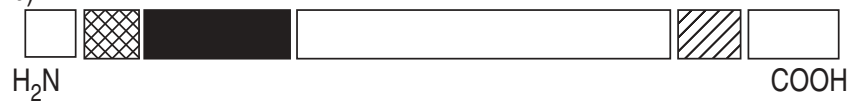

Fig. 5.-a) Western blot analysis of bronchoalveolar lavage (BAL) fluid of the index patient. Consistent with the strong immunostaining of the intra-alveolar-accumulated material for CTERMSP-C, antiCTERMSP-C identified aberrant $11-$ and $13-\mathrm{kDa}$ precursors of prosurfactant protein (SP)-C in the BAL fluid (lane 1). Only trace amounts of a $15-\mathrm{kDa}$ precursor of proSP-C were detected by antiNPROSP-C (lane 2). The presence of both monomeric 4-kDa and dimeric $8-\mathrm{kDa}$ SP-C in the BAL fluid (lane 3) provides indirect evidence that the heterozygous SP-C gene missense mutation (g.1286T $>$ C $($ I73T)) does not have a dominant negative effect on SP$\mathrm{C}$ biosynthesis. Furthermore, consistent with the immunostaining pattern, a 23-kDa precursor of SP-B (lane 4) and a mature dimeric $18-\mathrm{kDa}$ SP-B (lane 5) were found in the BAL fluid. b) Schematic diagram of SP-C. Specific polyclonal antibodies were generated against the C-terminal epitope Gly ${ }^{162}-$ Gly $^{174}$ (CTERMSP-C; $\mathbb{C}$ ) and the N-terminal epitope $\mathrm{Met}^{11}-\mathrm{Glu}^{23}$ (NPROSP-C; $\mathbf{0}$ : mature SP-C.

over a period of several years. The lavages, which were initially done on a monthly or bi-monthly basis, led to significant acute improvements of gas exchange with a reduced need for additional oxygen. This effect was most prominent during the initial 2 yrs of treatment and then waned. Therefore, additional glucocorticosteroid pulse therapy, together with azathioprin, was used over several years. However, a dependence on oxygen via nasal prongs $\left(2-5 \mathrm{~L} \cdot \mathrm{min}^{-1}\right)$ remained and growth was delayed. Using a high-calorie diet, near-normal weight gain was achieved. The patient's psychomotor development has been judged as appropriate up to the present age of $5 \mathrm{yrs}$.

\section{Discussion}

In the present study, the current authors have characterised the clinical, pathological and biochemical phenotype of a de novo heterozygous missense mutation of the SFTPC gene (g.1286T $>$ C) leading to the I73T substitution in proSP-C. This mutation is associated clinically with a severe respiratory insufficiency, histologically with combined patterns of NSIP and PAP, and biochemically with an abnormal trafficking of proSP-C and an intra-alveolar accumulation of aberrantly processed proSP-C. The histopathological and biochemical features, as seen with the g. 1286T $>C$ (I73T) mutation, are in contrast with that described for all previously reported mutations of either SFTPC or SFTPB [5, 7, 13, 14] (table 4). Thus, the g.1286T $>$ C (I73T) mutation underlies a novel genetic entity of chronic interstitial lung disease.

For several reasons, it is unlikely that the reported genetic change is a polymorphism or a rare variant. First, it results in the non-neutral substitution of a threonine for a highly
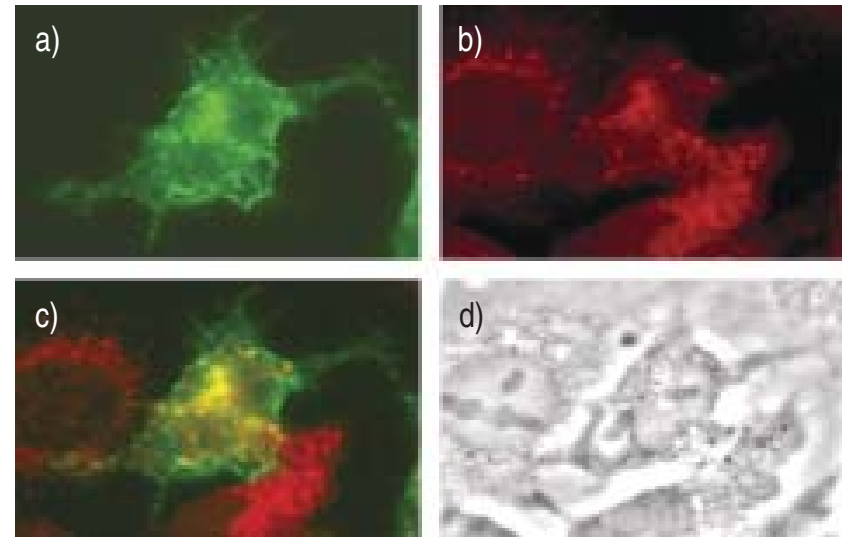

Fig. 6. - Enhanced green fluorescence protein (EGFP)/pro-surfactant (SP)-C $\mathrm{C}^{\mathrm{I} 73 \mathrm{~T}}$ fusion protein is directed to early endosome antigen (EEA)-1-positive cytoplasmic vesicles. A549 cells were transfected with EGFP/proSP-C ${ }^{1-197}$ or EGFP/proSP-C ${ }^{173 T}$ as described in the Materials and methods section. At $48 \mathrm{~h}$ after transfection, cells were fixed, permeabilised and stained with primary monoclonal EEA-1 antiserum and immunoglobulin G-specific secondary Texas Red-labelled antisera. Images acquired by video fluorescence microscopy using a high Q fluorescein isothiocyanate filter for EGFP and a Texas Red filter are representative of six separate experiments and $>50$ cells for each construct. The majority of a) EGFP/proSP-C ${ }^{173 T}$ co-localised with b) EEA-1 staining, as shown in (c). The phase image is shown in (d).

conserved isoleucine in the C-terminal propeptide and was not found among 100 unrelated Caucasian individuals. Secondly, the mutation was inherited de novo since nonpaternity or non-maternity were deemed unlikely (relatedness test, table 3), which was consistent with the dominant inheritance pattern as observed in two previously published SFTPC missense mutations [13, 14]. Interestingly, this genetic change appears to have occurred on the paternal allele, consistent with both the fact that it is a circumscribed lesion (point mutation) and advanced paternal age at conception (37.5 yrs). This point has been reviewed recently [27]. Thirdly, immunoassays performed on lung tissue and BAL fluid from the patient, as well as an in vitro functional assay, further established the deleterious consequences of this mutation.

A similar morphological picture has been described in infants with a severe and potentially lethal form of interstitial lung disease, known as chronic pneumonitis of infancy (CPI) [28]. CPI is commonly seen in full-term babies who are healthy at birth, but develop respiratory distress between 2 weeks and several months of age, and show interstitial infiltrates on chest radiography [29]. Since a finely granular eosinophilic material was found to fill many alveoli in the current index patient, in contrast to the patchy appearance (encompassing only a small percentage of the alveoli) in CPI [28], and the levels of SP-A in the BAL fluid were strongly increased, pulmonary alveolar proteinosis and hereditary SP-B deficiency were regarded as important differential diagnoses.

In line with increased levels of surfactant proteins in alveolar proteinosis lavage effluents [30-33], the current authors found a strong staining of the intra-alveolaraccumulated material for SP-A, precursors of SP-B, mature SP-B and SP-D, but not for CTERMSP-C, in two full-term babies with cryptogenic congenital PAP (fig. 4), and 26 adult patients with idiopathic and secondary PAP (unpublished data). Despite histopathological and immunohistochemical features of PAP, as well as increased levels of SP-A in BAL fluids, the combined patterns of NSIP and PAP, as well as the intra-alveolar accumulation of CTERMSP-C forms, are distinct features associated with the g.1286T $>C$ (I73T) missense mutation in the SFTPC gene. 
Table 4.-Genotype/phenotype correlation in patients with surfactant protein C gene (SFTPC) mutations

\begin{tabular}{|c|c|c|c|}
\hline & Index patient & NoGEE et al. [13] & THOMAS et al. [14] \\
\hline Genotype & $\begin{array}{c}\text { De novo heterozygous } \\
\text { g.1286T }>\text { C }(\mathrm{I} 73 \mathrm{~T}) \\
\text { SFTPC mutation }\end{array}$ & $\begin{array}{l}\text { Familial heterozygous } \\
\text { g.1727G }>\text { A } \\
\text { (deletion of exon 4) } \\
\text { SFTPC mutations }\end{array}$ & $\begin{array}{c}\text { Familial heterozygous } \\
\text { g.2188T }>\text { A (L188Q) } \\
\text { SFTPC mutations }\end{array}$ \\
\hline Phenotype & $\begin{array}{l}\text { Full-term infant with } \\
\text { combined histological } \\
\text { patterns of NSIP and PAP } \\
\text { Intra-alveolar accumulation } \\
\text { of aberrantly } \\
\text { processed proSP-C } \\
\text { Mono- and dimeric SP-C } \\
\text { forms in BAL fluids }\end{array}$ & $\begin{array}{l}\text { Full-term infant with } \\
\text { NSIP pattern and } \\
\text { mother with UIP pattern } \\
\text { Aberrant intracellular } \\
\text { localisation of } \\
\text { precursors of SP-C } \\
\text { Lack of SP-C in BAL fluids }\end{array}$ & $\begin{array}{l}\text { Kindred with NSIP pattern } \\
\text { in infants and } \\
\text { UIP pattern in adults } \\
\text { Aberrant intracellular } \\
\text { localisation of } \\
\text { precursors of SP-C }\end{array}$ \\
\hline $\begin{array}{l}\text { Trafficking of mutant } \\
\text { SP-C in A549 cells }\end{array}$ & $\begin{array}{l}\text { Intracellular routing to early } \\
\text { endosomes }\end{array}$ & $\begin{array}{l}\text { Formation of intracellular } \\
\text { aggregates [24] }\end{array}$ & $\begin{array}{l}\text { Formation of intracellular } \\
\text { aggregates }\end{array}$ \\
\hline
\end{tabular}

SP-C: surfactant protein C; NSIP: nonspecific interstitial pneumonia; PAP: pulmonary alveolar proteinosis; BAL: bronchoalveolar lavage; UIP: usual interstitial pneumonia. ${ }^{\#}$ : unpublished observation (M.F. Beers).

Hereditary SP-B deficiency, with over 20 different causative mutations of SFTPB, is a well-established cause of severe respiratory failure in newborns $[3,5,7]$. Immunohistochemically, the eosinophilic and PAS-positive material filling many alveoli in hereditary SP-B deficiency stains strongly for SP-A, precursors of SP-C and SP-D, but not SP-B [4]. In the index patient, an SFTPB mutation had been excluded and precursors of SP-B, as well as mature SP-B, were detectable by immunohistochemistry and Western-blot analysis of BAL fluids. Furthermore, the biochemical and immunohistochemical profiles of SP-C precursors of the patient with the g.1286T $>$ C (I73T) mutation contrasted with those observed for SP-B-deficient samples (fig. 4). Both a fragment of the $\mathrm{N}$-terminal propeptide of proSP-B and mature SP-B are necessary for the routing of precursors of SP-C to composite and lamellar bodies, where the final remodelling of the $\mathrm{N}$-terminus takes place $[18,20,34,35]$. In line with biochemical data, the strong staining of the intra-alveolaraccumulated material for NPROSP-C in SP-B-deficient samples is consistent with an intra-alveolar accumulation of a partially processed proSP-C (6-10 kDa), which contains residual N-terminal propeptide epitopes (fig. 4) [6]. In contrast, in the index patient, large amounts of CTERMSP-C forms accumulated in the alveoli and were identified as strong bands of 11 and $13 \mathrm{kDa}$, in an order of magnitude that is out of proportion to the minimal level of a single NPROSP-C form $(15 \mathrm{kDa})$ as seen in the BAL fluid. The results here are consistent with an intra-alveolar accumulation of proteins containing the C-terminal propeptide domain that, however, does not correlate with the sizes of the known processing intermediates of proSP-C in type-II pneumocytes [18, 36].

Two different inherited heterozygous mutations of the SFTPC gene have been previously identified and shown to associate with respiratory insufficiency and interstitial lung disease; the former in a term-born baby girl (mother and maternal grandfather also affected) and the latter in a multigenerational kindred $[13,14]$. These familial mutations were accompanied by NSIP and DIP in babies or children, and UIP in affected adults [13]. However, no PAP-like features were observed in lung-biopsy specimens of these patients $[13,14]$. Furthermore, the staining pattern for precursors of SP-C associated with the g.1286T $>$ C (I73T) mutation is different from that of the g.1727G $>$ A (deletion of exon 4) and g.2188T $>$ A (L188Q) SFTPC mutations that were associated with an abnormal intracellular, but not intra-alveolar, localisation of precursors of SP-C and a lack of mature SP-C [13, 14] (table 4).

SP-C is a monomeric dipalmitoylated protein with a molecular mass of $4.2 \mathrm{kDa}$, which is synthesised exclusively by type-II pneumocytes as a $21-\mathrm{kDa}$ propeptide (proSP-C). En route from its site of synthesis to the lamellar bodies via the endoplasmic reticulum, the Golgi apparatus and multivesicular bodies, proSP-C undergoes extensive C-and N-terminal post-translational processing [18, 19]. Transfection studies confirmed that both mutations, g.1727G $>$ A (deletion of exon 4) and g.2188T $>$ A (L188Q), have dominant negative effects on SP-C biosynthesis that leads to misfolding and trapping of proSP-C or formation of aggresomes in type-II pneumocytes and A549 cells $[14,24]$. To study the consequences of the g.1286T $>$ C (I73T) missense mutation on intracellular trafficking of proSP-C, the current authors generated an EGFP/ proSP-C $C^{173 T}$ fusion protein with the aim to follow the biosynthetic routing in vitro. While an EGFP/wild type proSP-C $C^{1-197}$ fusion is constantly targeted to CD63(+), EEA1(-) vesicles [23, 24, 37], the EGFP/proSP-C ${ }^{\mathrm{I} 73 \mathrm{~T}}$ protein product localised to EEA1(+) vesicles in A549 cells, consistent with an abnormal trafficking of the mutant proprotein to an endosomal compartment.

Most data indicate that proSP-C is an integral membrane protein inserted into membranes, in a type-II transmembrane configuration with the C-terminus of proSP-C, inside the lumen of the endoplasmic reticulum and the Golgi vesicles [18, 38, 39]. Under physiological conditions, processing of proSP-C occurs along the regulated secretory pathway in multivesicular and lamellar bodies, after the reconfiguration (inversion) of the transmembrane orientation during fusion events with the target compartment $[18,20]$. In the case of I73T, the mutant product is directed to the constitutive pathway and remains membrane-associated in a type-II orientation. The 11- and 13-kDa CTERMSP-C forms in the BAL fluid may be due to a nonspecific cleavage of the $\mathrm{COOH}$ terminus of the mutant proSP-C in the vesicle lumen. During fusion of the constitutive vesicle with the plasma membrane, the lumenal contents are secreted, leading to detectable CTERMSP-C forms in the BAL fluid. In line with an abnormal trafficking and fusion of the constitutive vesicles with the plasma membrane, the current authors detected abnormal vesicular organelles with an electron-dense core in the process of being secreted by type-II pneumocytes.

It is generally accepted that under physiological conditions both SP-B and SP-C are delivered together by multivesicular bodies to lamellar bodies and secreted with the lamellar bodies in the alveoli [40, 41]. Since the current authors detected not only CTERMSP-C forms, but also precursors of $\mathrm{SP}-\mathrm{B}$, in the alveoli of the index patient, a protein-protein interaction of mutant proSP-C and wild-type proSP-B may be possible. However, despite a misfolding and trapping of 
proSP-C or formation of aggresomes in type-II pneumocytes, and a lack of mature SP-C in patients with g.1727G $>$ A (deletion of exon 4) or g.2188T $>$ A (L188Q) SFTPC mutations, no abnormal intracellular distribution of proSP-B or a lack of mature SP-B was reported [13, 14]. Furthermore, the current authors also observed an intra-alveolar accumulation of precursors of SP-B in cryptogenic congenital PAP, idiopathic and secondary PAP, as well as Pneumocystis carinii pneumonia (unpublished data). Therefore, an intra-alveolar accumulation of precursors of SP-B occurs under various pathological conditions and may not be due to a specific protein-protein interaction of mutant proSP-C and wild-type proSP-B.

Genetically engineered mice, as well as newborn calves of the Belgian White and Blue breed, with a lack or deficiency of SP-C, develop interstitial lung disease during postnatal growth [13, 42, 43]. However, anti-SP-C readily identified mature mono- and dimeric SP-C forms in BAL fluids from the index patient, providing indirect evidence that the heterozygous g.1286T $>C$ (I73T) missense mutation did not have a dominant-negative effect on SP-C biosynthesis. Previously, the current authors showed that heterotypic oligomerisation of wild-type and mutant proSP-C provides the molecular mechanism for the dominant-negative effect on SP-C biosynthesis of the g.1727G $>$ A (deletion of exon 4) mutation [24]. In case of the g.1286T $>C$ (I73T) missense mutation, the substitution of a threonine for an isoleucine in the C-terminal propeptide may affect the oligomerisation process, and thus prevent heterotypic oligomerisation that is responsible for the dominant-negative effect on SP-C biosynthesis. Therefore, the mature mono- and dimeric SP$\mathrm{C}$ forms detected in BAL fluids might be contributed from wild-type proSP-C that is regularly processed along the regulated secretory pathway.

Dimeric SP-C forms of $\sim 7-9 \mathrm{kDa}$ consisting of non- or monopalmitoylated SP-C have been found in adult patients suffering from PAP [44 46]. Palmitoylation of proSP-C does not appear to be related to proprotein targeting, as substitution of the cystein residues normally undergoing palmitoylation did not influence the sorting of the protein [39]. However, dimeric SP-C may be causally related to the intraalveolar accumulation of surfactant proteins, since it is cleared from lungs with an increased half-life, it is not removed from alveolar macrophages, and it hinders clearance of SP-B and monomeric SP-C by alveolar macrophages. Furthermore, dimeric SP-C is toxic to alveolar macrophages through an increased formation of reactive oxygen species $[45,46]$. Importantly, the pathology observed here cannot be attributed to a lack of mature SP-C, but rather resulted from a deleterious gain of function induced by expression products of the mutant allele. In contrast to g. $1727 \mathrm{G}>\mathrm{A}$ (deletion of exon 4) and g.2188T $>$ A (L188Q) SFTPC mutations [13, 14], g.1286T $>C$ (I73T) represents a new class of SFTPC mutations, which is marked by a distinct trafficking, processing, palmitoylation, and secretion of the mutant and wild-type SP-C.

Based on the histopathological and biochemical findings of an intra-alveolar accumulation of aberrant surfactant proteins, the therapeutical approach for this patient was guided by the known clinical efficacy of repetitive whole lung lavages in adult patients with idiopathic PAP, with the aim to remove the accumulated proteinaceous material from the alveolar space. The efficacy of the lavages was clearly seen by significant acute improvements of gas exchange, with a reduced need for additional oxygen. This therapeutic efficacy extended from several days to several weeks. Since this effect waned after 2 yrs of treatment, an additional glucocorticosteroid pulse therapy, together with azathioprin, was initiated. The rationale of anti-inflammatory treatment with systemic steroids and azathioprine was not based on earlier experience from patients with other mutations of SFTPC or SFTPB, but was based on the histology, i.e. to target the mild chronic interstitial inflammation observed. Combined therapies appeared helpful since they were met by adequate physical and psychosocial development of the affected child. However, the contribution of the anti-inflammatory treatment cannot be unequivocally determined, since the natural course of patients with SFTPC mutations may be very variable. Furthermore, it must be kept in mind that this was observed in a single patient and that the phenotype of patients with other SFTPC mutations may be different. Therefore, followup studies are necessary to characterise the natural course of the disease and to evaluate the efficacy of therapeutic approaches.

In conclusion, the current authors report a de novo missense mutation of the surfactant protein $\mathrm{C}$ gene, which is associated with combined histological patterns of nonspecific interstitial pneumonia and pulmonary alveolar proteinosis, and biochemically with an abnormal trafficking and intra-alveolar accumulation of aberrantly processed pro-surfactant protein C. This report heralds the emerging diversity of phenotypes associated with the expression of mutant surfactant protein $\mathrm{C}$ proteins, and highlights the need for a careful clinical, pathological, and molecular characterisation of the patients.

Acknowledgements. The excellent technical assistance of S. Freese, S. Geiger, A. Gerken, H. Hühn, M. Kochem, S. Marque, O.Pascaud, U. Thomek and S.J. Russo is highly appreciated.

\section{References}

1. Hawgood S. Pulmonary surfactant apoproteins: a review of protein and genomic structure. Am J Physiol 1989; 257: L13L22.

2. Batenburg JJ, Haagsman HP. The lipids of pulmonary surfactant: dynamics and interactions with proteins. Prog Lipid Res 1998; 37: 235-276.

3. Nogee LM, de Mello DE, Dehner LP, Colten HR. Brief report: deficiency of pulmonary surfactant protein $\mathrm{B}$ in congenital alveolar proteinosis. N Engl J Med 1993; 328: 406-410.

4. deMello DE, Nogee LM, Heyman S, et al. Molecular and phenotypic variability in the congenital alveolar proteinosis syndrome associated with inherited surfactant protein B deficiency. J Pediatr 1994; 125: 43-50.

5. Tredano M, Griese M, De Blic J, et al. Analysis of 40 sporadic or familial neonatal and pediatric cases with severe unexplained respiratory distress: relationship to SFTPB. Am $J$ Med Genet 2003; 119A: 324-339.

6. Beers MF, Hamvas A, Moxley MA, et al. Pulmonary surfactant metabolism in infants lacking surfactant protein B. Am J Respir Cell Mol Biol 2000; 22: 380-391.

7. Nogee LM, Wert SE, Proffit SA, Hull WM, Whitsett JA. Allelic heterogeneity in hereditary surfactant protein B (SPB) deficiency. Am J Respir Crit Care Med 2000; 161: 973981.

8. Rosen SH, Castleman B, Liebow AA. Pulmonary alveolar proteinosis. N Engl J Med 1958; 258: 1123-1142.

9. Kitamura $\mathrm{T}$, Tanaka $\mathrm{N}$, Watanabe $\mathrm{J}$, et al. Idiopathic pulmonary alveolar proteinosis as an autoimmune disease with neutralizing antibody against granulocyte/macrophage colony-stimulating factor. J Exp Med 1999; 190: 875-880.

10. Seymour JF, Presneill JJ. Pulmonary alveolar proteinosis: progress in the first 44 years. Am J Respir Crit Care Med 2002; 166: 215-235.

11. American Thoracic Society/European Respiratory Society international multidisciplinary consensus classification of the idiopathic interstitial pneumonias. This joint statement of the 
American Thoracic Society (ATS), and the European Respiratory Society (ERS) was adopted by the ATS board of directors, June 2001 and by the ERS Executive Committee, June 2001. Am J Respir Crit Care Med 2002; 165: 277-304.

12. Marshall RP, Puddicombe A, Cookson WO, Laurent GJ. Adult familial cryptogenic fibrosing alveolitis in the United Kingdom. Thorax 2000; 55: 143-146.

13. Nogee LM, Dunbar AE 3rd, Wert SE, Askin F, Hamvas A, Whitsett JA. A mutation in the surfactant protein $\mathrm{C}$ gene associated with familial interstitial lung disease. $N$ Engl $J$ Med 2001; 344: 573-579.

14. Thomas AQ, Lane K, Phillips J 3rd, et al. Heterozygosity for a surfactant protein $\mathrm{C}$ gene mutation associated with usual interstitial pneumonitis and cellular nonspecific interstitial pneumonitis in one kindred. Am J Respir Crit Care Med 2002; 165: 1322-1328.

15. Uchida K, Nakata K, Trapnell BC, et al. High-affinity autoantibodies specifically eliminate granulocyte-macrophage colony-stimulating factor activity in the lungs of patients with idiopathic pulmonary alveolar proteinosis. Blood 2004; 103: 1089-1098.

16. Kitamura $\mathrm{T}$, Uchida $\mathrm{K}$, Tanaka $\mathrm{N}$, et al. Serological diagnosis of idiopathic pulmonary alveolar proteinosis. $\mathrm{Am}$ J Respir Crit Care Med 2000; 162: 658-662.

17. Glasser SW, Korfhagen TR, Perme CM, Pilot MT, Kister SE, Whitsett JA. Two SP-C genes encoding human pulmonary surfactant proteolipid. J Biol Chem 1988; 263: 10326-10331.

18. Johnson AL, Braidotti P, Pietra GG, et al. Post-translational processing of surfactant protein-C proprotein: targeting motifs in the $\mathrm{NH}(2)$-terminal flanking domain are cleaved in late compartments. Am J Respir Cell Mol Biol 2001; 24: 253-263.

19. Solarin KO, Ballard PL, Guttentag SH, Lomax CA, Beers MF. Expression and glucocorticoid regulation of surfactant protein C in human fetal lung. Pediatr Res 1997; 42: 356-364.

20. Brasch F, Ten Brinke A, Johnen G, et al. Involvement of cathepsin $\mathrm{H}$ in the processing of the hydrophobic surfactantassociated protein $\mathrm{C}$ in type II pneumocytes. Am $J$ Respir Cell Mol Biol 2002; 26: 659-670.

21. Bornhorst B, Stuhrmann M, Nogee LM, Brasch F, Müller $\mathrm{KM}$, Poets CF. Congentital pulmonary alveolar proteinosis - hereditary deficiency of surfactant protein B (Kongenitale alveolarproteinose: hereditärer mangel an surfactant-protein B). Monatsschrift der Kinderheilkunde 1996; 144: 1214-1217.

22. Griese M, Birrer P, Demirsoy A. Pulmonary surfactant in cystic fibrosis. Eur Respir J 1997; 10: 1983-1988.

23. Wang WJ, Russo SJ, Mulugeta S, Beers MF. Biosynthesis of surfactant protein C (SP-C). Sorting of SP-C proprotein involves homomeric association via a signal anchor domain. J Biol Chem 2002; 277: 19929-19937.

24. Wang WJ, Mulugeta S, Russo SJ, Beers MF. Deletion of exon 4 from human surfactant protein $\mathrm{C}$ results in aggresome formation and generation of a dominant negative. J Cell Sci 2003; 116: 683-692.

25. Russo SJ, Wang W, Lomax CA, Beers MF. Structural requirements for intracellular targeting of SP-C proprotein. Am J Physiol 1999; 277: L1034-L1044.

26. Griese M, Maderlechner N, Ahrens P, Kitz R. Surfactant proteins $\mathrm{A}$ and $\mathrm{D}$ in children with pulmonary disease due to gastroesophageal reflux. Am J Respir Crit Care Med 2002; 165: 1546-1550.

27. Hurst L, Ellegren H. Mystery of the mutagenic male. Nature 2002; 420: 356-365.

28. Katzenstein AL, Gordon LP, Oliphant M, Swender PT. Chronic pneumonitis of infancy. A unique form of interstitial lung disease occurring in early childhood. Am J Surg Pathol 1995; 19: 439-447.
29. Langston C. Pediatric Lung Biopsy. Cagle PT. Diagnostic Pulmonary Pathology. In: Cagle PT, ed. Lung Biology in Health and Disease. New York, Marcel Decker Inc, 2000; pp. 19-46.

30. Doyle IR, Davidson KG, Barr HA, Nicholas TE, Payne K, Pfitzner J. Quantity and structure of surfactant proteins vary among patients with alveolar proteinosis. Am J Respir Crit Care Med 1998; 157: 658-664.

31. Crouch E, Persson A, Chang D. Accumulation of surfactant protein $\mathrm{D}$ in human pulmonary alveolar proteinosis. $\mathrm{Am}$ J Pathol 1993; 142: 241-248.

32. Alberti A, Luisetti M, Braschi A, et al. Bronchoalveolar lavage fluid composition in alveolar proteinosis. Early changes after therapeutic lavage. Am J Respir Crit Care Med 1996; 154: 817-820.

33. Honda Y, Takahashi H, Shijubo N, Kuroki Y, Akino T. Surfactant protein-A concentration in bronchoalveolar lavage fluids of patients with pulmonary alveolar proteinosis. Chest 1993; 103: 496-499.

34. Lin S, Akinbi HT, Breslin JS, Weaver TE. Structural requirements for targeting of surfactant protein B (SP-B) to secretory granules in vitro and in vivo. J Biol Chem 1996; 271: 19689-19695.

35. Lin S, Phillips KS, Wilder MR, Weaver TE. Structural requirements for intracellular transport of pulmonary surfactant protein B (SP-B). Biochim Biophys Acta 1996; 1312: 177-185.

36. Beers MF, Kim CY, Dodia C, Fisher AB. Localization, synthesis, and processing of surfactant protein SP-C in rat lung analyzed by epitope-specific antipeptide antibodies. J Biol Chem 1994; 269: 20318-20328.

37. Kabore AF, Wang WJ, Russo SJ, Beers MF. Biosynthesis of surfactant protein $\mathrm{C}$ : characterization of aggresome formation by EGFP chimeras containing propeptide mutants lacking conserved cysteine residues. J Cell Sci 2001; 114: 293-302.

38. Keller A, Eistetter HR, Voss T, Schafer KP. The pulmonary surfactant protein C (SP-C) precursor is a type II transmembrane protein. Biochem J 1991; 277: 493-499.

39. Ten Brinke A, Batenburg JJ, Gadella BM, Haagsman HP, Vaandrager AB, van Golde LM. The juxtamembrane lysine and arginine residues of surfactant protein $\mathrm{C}$ precursor influence palmitoylation via effects on trafficking. $\mathrm{Am}$ J Respir Cell Mol Biol 2001; 25: 156-163.

40. Weaver TE, Conkright JJ. Function of surfactant proteins B and C. Annu Rev Physiol 2001; 63: 555-578.

41. Voorhout WF, Weaver TE, Haagsman HP, Geuze HJ, van Golde LM. Biosynthetic routing of pulmonary surfactant proteins in alveolar type II cells. Microsc Res Tech 1993; 26: 366-373.

42. Glasser SW, Burhans MS, Korfhagen TR, et al. Altered stability of pulmonary surfactant in SP-C-deficient mice. Proc Natl Acad Sci USA 2001; 98: 6366-6371.

43. Danlois F, Zaltash S, Johansson J, et al. Very low surfactant protein $\mathrm{C}$ contents in newborn Belgian white and blue calves with respiratory distress syndrome. Biochem $J$ 2000; 351: 779-787.

44. Voss T, Schafer KP, Nielsen PF, et al. Primary structure differences of human surfactant-associated proteins isolated from normal and proteinosis lung. Biochim Biophys Acta 1992; 1138: 261-267.

45. Li ZY, Suzuki Y, Kurozumi M, Shen HQ, Duan CX. Removal of a dimeric form of surfactant protein $\mathrm{C}$ from mouse lungs: its acceleration by reduction. J Appl Physiol 1998; 84: 471-478.

46. Shen HQ, Duan CX, Li ZY, Suzuki Y. Effects of proteinosis surfactant proteins on the viability of rat alveolar macrophages. Am J Respir Crit Care Med 1997; 156: 16791687. 